BMJ Open

Sport \&

Exercise

Medicine

\title{
ACL reconstruction with hamstring tendon autograft and accelerated brace- free rehabilitation: a systematic review of clinical outcomes
}

\author{
Rob P A Janssen, ${ }^{1}$ Nicky van Melick, ${ }^{2}$ Jan B A van Mourik, ${ }^{1}$ Max Reijman, ${ }^{1,3}$ \\ Lodewijk W van Rhijn ${ }^{4}$
}

To cite: Janssen RPA, van Melick N, van Mourik JBA et al. ACL reconstruction with hamstring tendon autograft and accelerated brace-free rehabilitation: a systematic review of clinical outcomes. BMJ Open Sport \& Exercise Medicine 2018;4:e000301. doi:10.1136/ bmjsem-2017-000301

Parts of the abstract of this paper have been presented at the 17th ESSKA Congress (4-7 May 2016) in Barcelona, Spain, as a poster presentation with interim findings. The poster's abstract was published in 'Poster Abstracts' in Knee Surgery Sports Traumatology Arthroscopy.

Accepted 19 February 2018

Check for updates

${ }^{1}$ Orthopaedic Center Máxima, Máxima Medical Center, Eindhoven, The Netherlands ${ }^{2}$ Knee Expert Center Eindhoven, Eindhoven, The Netherlands ${ }^{3}$ Department of Orthopaedic Surgery, Erasmus Medical Center, University Medical Center Rotterdam, Rotterdam, The Netherlands

${ }^{4}$ Orthopaedic Surgery \& Traumatology, Maastricht University Medical Center, Maastricht, The Netherlands

Correspondence to Dr Rob P A Janssen, ; r. janssen@mmc.nl

\section{ABSTRACT}

Objective To investigate the clinical outcomes after hamstring tendon autograft $A C L$ reconstruction (ACLR) with accelerated, brace-free rehabilitation.

Design Systematic review according to Preferred Reporting Items for Systematic Reviews and Meta-Analysis guidelines.

Data sources Embase, MEDLINE Ovid, Web of Science, Cochrane CENTRAL and Google scholar from 1 January 1974 to 31 January 2017.

Eligibility criteria for selecting studies Study designs reporting outcomes in adults after arthroscopic, primary ACLR with hamstring autograft and accelerated, brace-free rehabilitation.

Results Twenty-four studies were included in the review. The clinical outcomes after hamstring tendon autograft ACLR with accelerated brace-free rehabilitation were the following: (1) early start of open kinetic exercises at 4 weeks in a limited range of motion (ROM, $90^{\circ}-45^{\circ}$ ) and progressive concentric and eccentric exercises from 12 weeks did not alter outcomes, (2) gender and age did not influence clinical outcomes, (3) anatomical reconstructions showed better results than non-anatomical reconstructions, (4) there was no difference between single-bundle and double-bundle reconstructions, (5) femoral and tibial tunnel widening occurred, (6) hamstring tendons regenerated after harvest and (7) biological knowledge did not support return to sports at 4-6 months. Conclusions After hamstring tendon autograft ACLR with accelerated brace-free rehabilitation, clinical outcome is similar after single-bundle and double-bundle ACLR. Early start of open kinetic exercises at 4 weeks in a limited ROM $\left(90^{\circ}-45^{\circ}\right)$ and progressive concentric and eccentric exercises from 12 weeks postsurgery do not alter clinical outcome. Further research should focus on achievement of best balance between graft loading and graft healing in the various rehabilitation phases after ACLR as well as on validated, criterion-based assessments for safe return to sports.

Level of evidence Level 2b; therapeutic outcome studies.

\section{INTRODUCTION}

Rehabilitation after ACL reconstruction (ACLR) could be described as adaptations

\section{What is already known?}

- Accelerated rehabilitation, defined as earlyunrestricted motion, immediate weight-bearing and eliminating the use of immobilising braces, is appropriate after $\mathrm{ACL}$ reconstruction with patellar tendon grafts.

- Advantages of accelerated, brace-free rehabilitation protocols after ACL reconstruction are earlier normal function of the knee and immediate weight-bearing postsurgery.

- Hamstring tendon ACL autografts undergo an intraarticular remodelling process.

\section{What are the new findings?}

After hamstring tendon autograft ACL reconstruction with accelerated brace-free rehabilitation:

- Strong evidence suggests that clinical outcome is similar after single-bundle and double-bundle ACL reconstruction.

- Moderate evidence suggests that early start of open kinetic exercises at 4 weeks in a limited range of motion $\left(90^{\circ}-45^{\circ}\right)$ and progressive concentric and eccentric exercises from 12 weeks postsurgery do not alter clinical outcome.

to a complex biological system. ${ }^{1}$ Outcomes after ACLR are influenced by both surgical and rehabilitation factors. ACL surgery requires the understanding of several factors: anatomical graft placement, mechanical properties of the selected graft tissue, mechanical behaviour and fixation strength of fixation materials as well as the biological processes that occur during graft remodelling, maturation and incorporation. ${ }^{1-5}$ These factors influence directly the mechanical properties of the knee joint after ACLR and should, in combination with rehabilitation progress, dictate the time course until normal function of the knee joint can be expected. ${ }^{56}$ 
After surgery, graft healing is characterised by a remodelling process. ${ }^{2} 3589$ During this period, the graft will undergo changes, becoming morphologically similar to intact ligament tissue. ${ }^{259-11}$ Contemporary rehabilitation-defined as early-unrestricted motion, immediate weight-bearing and eliminating the use of immobilising braces-is appropriate after ACLR with patellar tendon grafts. ${ }^{12-20}$ However, conclusions are unclear when evaluating the effects of this type of rehabilitation after hamstring autograft ACLR. ${ }^{1}$ This is important because the hamstring tendons are a popular graft source for ACLR. ${ }^{7}$ Advantages of accelerated, brace-free rehabilitation protocols after ACLR are earlier normal function of the knee, weight-bearing and alleged ability to return to even most strenuous activities after primary ACLR at 6 months. ${ }^{25101521-26}$ A major challenge in postoperative rehabilitation after ACLR is optimising the balance between muscular strengthening exercises and loading of the graft to stimulate graft cells to produce cellular and extracellular components for the preservation of graft stability, without compromising graft integrity, which might result into an early elongation of the ACLR. ${ }^{2511} 152728$

The purpose of this systematic review is to present the current knowledge on outcomes after hamstring tendon autograft ACLR with accelerated, brace-free rehabilitation in adults. The primary aim was to examine the influence of different rehabilitation protocols, patient characteristics and surgical techniques on clinical outcomes after hamstring tendon autograft ACLR. The secondary aim was to examine the influence of contemporary rehabilitation on tunnel widening, tendon regeneration and time to return to sports after hamstring tendon autograft ACLR.

\section{METHODS}

This systematic review was performed according to the Preferred Reporting Items for Systematic Reviews and Meta-Analysis (PRISMA). ${ }^{29}$ We had six key review questions:

1. How do differences in rehabilitation protocols affect clinical outcomes?

2. How do different patient characteristics affect clinical outcomes?

3. How do different non-anatomical and anatomical surgical techniques affect clinical outcomes?

4. Does accelerated, brace-free rehabilitation influence tunnel widening?

5. Do hamstring tendons regenerate after harvest?

6. Does the current biological knowledge on hamstring tendon autografts support early return to sports?

\section{Eligibility criteria}

The inclusion and exclusion criteria for the systematic review are presented in box 1 .

\section{Electronic search}

A systematic electronic search was performed using specific search terms in the following databases: Embase,

\section{Box 1 Inclusion and exclusion criteria}

\section{Inclusion criteria}

- Studies (meta-analysis, randomised, non-randomised, systematic reviews, case series, prospective or retrospective design) evaluating outcome in adult patients undergoing isolated ACL reconstruction (ACLR).

- Studies must have included an accelerated rehabilitation protocol. Accelerated rehabilitation is characterised by immediate postoperative weight-bearing, without restriction in motion and brace-free rehabilitation. Return to sports is allowed after 4-6 months.

- Any arthroscopic surgical method of primary intra-articular ACLR.

- Hamstring tendon autograft.

- Human in vivo studies with reported outcome.

- English language.

- Abstract and full text available.

\section{Exclusion criteria}

- Concomitant surgery limiting an accelerated rehabilitation protocol (meniscal repair or transplant, osteotomy, microfracture autologous cartilage implantation or matrix autologous chondrocyte implantation).

- Revision surgery.

- Allografts, bone-patellar tendon graft, quadriceps tendon or synthetic grafts.

- Multiligament reconstructions.

- Posterolateral, medial or posterior cruciate ligament instability.

- Non-defined rehabilitation protocol.

- Children and adolescents.

- Animal or cadaveric (in vitro) studies.

- Non-arthroscopic ACLR.

Non-English language.

Abstract or full text not available.

MEDLINE Ovid, Web of Science, Cochrane CENTRAL and Google scholar from 1 January 1974 to 31 January 2017 (online supplementary file 1).

\section{Study selection}

All potentially eligible articles were screened by title, abstract and full text by two teams of reviewers (RPAJ and NvM, and RPAJ and JBAvM). When two reviewers did not reach consensus, a third reviewer ( $\mathrm{NvM}$ or JBAvM) made the final decision. We screened the reference lists of excluded and included articles for potentially eligible articles that may have been missed in the electronic database search.

\section{Data extraction}

Data were extracted by two independent reviewers (RPAJ and NvM), and disagreements were resolved by consensus.

We extracted data on key variables regarding surgical techniques, graft type, patient demographics, details of rehabilitation, patient-reported outcome, clinical outcome measures and radiological evaluation.

\section{Synthesis of results}

Due to substantial heterogeneity with regard to surgical techniques, populations, outcome and study design, it was 
not possible to pool data for statistical analysis. Instead, we used a best-evidence synthesis ${ }^{30} 31$ with the following ranking of levels of evidence:

1. Strong evidence is provided by two or more studies with good quality (low risk of bias) and by generally consistent findings in all studies $(\geq 75 \%$ of the studies reported consistent findings).

2. Moderate evidence is provided by one good quality (low-risk of bias) study and two or more questionable quality (higher risk of bias) studies and by generally consistent findings in all studies $(\geq 75 \%)$.

3. Limited evidence is provided by one or more questionable quality (higher risk of bias) studies or one good quality (low-risk of bias) study and by generally consistent findings $(\geq 75 \%)$.

4. Conflicting evidence is provided by conflicting findings $(<75 \%$ of the studies reported consistent findings). ${ }^{31}$

\section{Risk of bias assessment}

Two reviewers (RPAJ and NvM) assessed the risk of bias of the articles independently. If the two reviewers did not reach consensus, a third reviewer (JBAvM) made the final decision. The reviewers were not blinded for author, journal or publication. The assessment of risk of bias of all articles was performed by standardised checklists of the Dutch Cochrane Library (www.netherlands.co chrane.org/beoordelingsformulieren-en-andere-downloads), namely for therapy and prevention (intervention, randomised controlled trials (RCTs)) and for prognosis (cohort studies).

The assessment of risk of bias for RCTs used nine criteria, displayed in table 1 . These nine items could be

Table 1 Cochrane criteria for the assessment of RCTs and cohort studies

\begin{tabular}{|c|c|}
\hline RCT & Cohort studies \\
\hline $\begin{array}{l}\text { 1. Is a method of } \\
\text { randomisation applied? }\end{array}$ & $\begin{array}{l}\text { 1. Are study groups clearly } \\
\text { defined? }\end{array}$ \\
\hline 2. Is randomisation blinded? & 2. Is there any selection bias? \\
\hline 3. Are the patients blinded? & $\begin{array}{l}\text { 3. Is the exposure clearly } \\
\text { defined? }\end{array}$ \\
\hline 4. Is the therapist blinded? & $\begin{array}{l}\text { 4. Is the outcome clearly } \\
\text { defined? }\end{array}$ \\
\hline $\begin{array}{l}\text { 5. Is the outcome assessor } \\
\text { blinded? }\end{array}$ & $\begin{array}{l}\text { 5. Is the outcome } \\
\text { assessment blinded? }\end{array}$ \\
\hline $\begin{array}{l}\text { 6. Are the groups } \\
\text { comparable? }\end{array}$ & 6. Is the follow-up accurate? \\
\hline $\begin{array}{l}\text { 7. Is there an acceptable } \\
\text { lost-to-follow-up? }\end{array}$ & $\begin{array}{l}\text { 7. Is there an acceptable } \\
\text { lost-to-follow-up? }\end{array}$ \\
\hline $\begin{array}{l}\text { 8. Is there an intention-to- } \\
\text { treat? }\end{array}$ & $\begin{array}{l}\text { 8. Are confounders described } \\
\text { and/or eliminated? }\end{array}$ \\
\hline $\begin{array}{l}\text { 9. Are treatments } \\
\text { comparable? }\end{array}$ & \\
\hline
\end{tabular}

$\mathrm{RCT}$, randomised controlled trial. rated 'yes' (+), 'no' (-) or 'do not know' (?). The same list was used for assessing clinical controlled trials, but these scored a 'no' for items 1 and 2.

The assessment of risk of bias for cohort studies described eight items, displayed in table 1 . All eight items could be rated positive $(+)$, negative $(-)$ or 'do not know' (?). The same list was used for cross-sectional studies, but these scored a '-' for item 2 because the study design could cause a selection bias.

We also evaluated two additional items due to their influence on outcome after ACLR and contemporary rehabilitation: (1) accurate description of the rehabilitation protocol and (2) ratio of men and women participating in the study. A final judgement of 'good', 'questionable' or 'poor' was given to every article. A 'good' was assigned to articles scoring positive for more than $50 \%$ of all items (low risk of bias); a 'questionable' if the positive score was between $30 \%$ and $50 \%$ (questionable risk of bias) and a 'poor' was assigned to articles with a positive score inferior to $30 \%$ (high risk of bias). The articles with a total score of 'good' and 'questionable' were included in the review.

\section{RESULTS}

\section{Study selection}

The PRISMA flow chart of the systematic review is presented in figure 1. A total of 29 studies were selected for the risk of bias assessment: 6 RCTs, ${ }^{10}{ }^{12} 32-354$ clinical controlled trials, ${ }^{36-39} 12$ prospective cohort studies, ${ }^{21}{ }^{40-50} 4$ cross-sectional studies, ${ }^{951-53}$ and 3 retrospective cohort studies. ${ }^{54-56}$

\section{Risk of bias assessment}

The results of the risk of bias assessment for the included studies are presented in tables 2 and 3. Five articles were discarded because of the total score 'poor' after quality appraisal. Twenty-four articles were included in the systematic review.

\section{Details of studies and rehabilitation}

The details of the included studies are presented in table 4 . The details of accelerated rehabilitation of the 24 included studies are presented in table 5 .

\section{Results of individual studies and answers to research questions}

How do differences in rehabilitation protocols affect clinical outcomes after hamstring tendon autograft ACLR with accelerated, brace-free rehabilitation?

Czaplicki $e t a l^{46}$ prospectively evaluated serial changes in isokinetic muscle strength preoperatively and postoperatively. They found significant differences between extension peak torques for the injured and healthy limbs at all stages of accelerated rehabilitation. At 1 year, there was still a deficit in muscle strength of the operated leg. ${ }^{46}$

The effects of accelerated brace-free free rehabilitation versus rehabilitation with brace and limited ROM for 4 weeks postsurgery were examined by Christensen $e t a .^{12}$ 


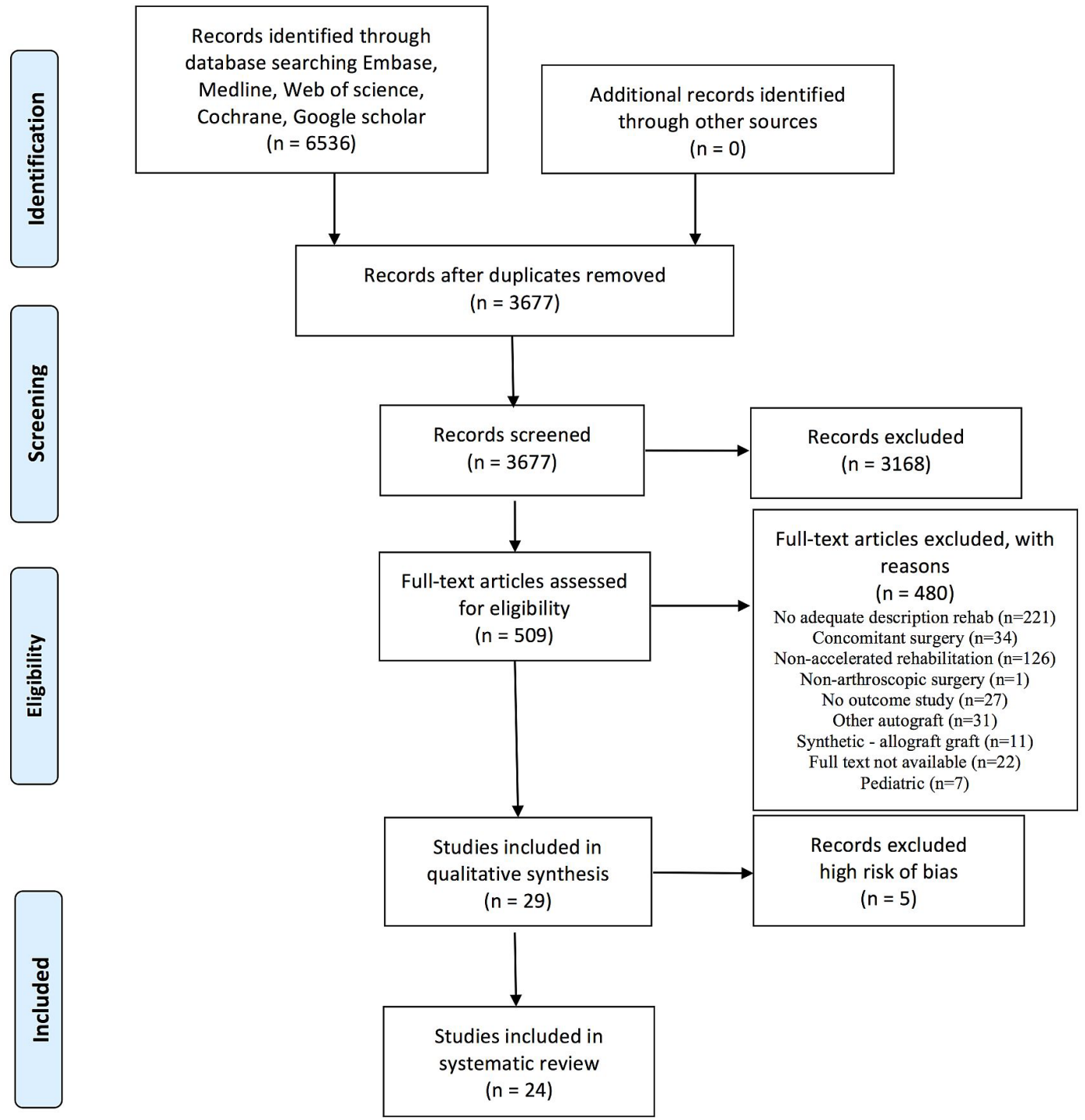

Figure 1 Preferred Reporting Items for Systematic Reviews and Meta-Analysis flow diagram. From: Moher D, Liberati A, Tetzlaff J, Altman DG. The PRISMA Group. Preferred items for systematic reviews and meta-analyses: the PRISMA statement. PLoS Med 2009;6(6):e1000097. doi:10.1371/journal.pmed1000097.

Table 2 Risk of bias assessment of RCTs and CCTs

\begin{tabular}{|c|c|c|c|c|c|c|c|c|c|c|c|c|c|}
\hline Article & $\begin{array}{l}\text { Study } \\
\text { design }\end{array}$ & 1 & 2 & 3 & 4 & 5 & 6 & 7 & 8 & 9 & $\begin{array}{l}\text { Accurate } \\
\text { description } \\
\text { rehabilitation }\end{array}$ & $\begin{array}{l}\text { Men- } \\
\text { women ratio }\end{array}$ & Total score \\
\hline Baltaci et $a l^{32}$ & RCT & + & + & - & $?$ & - & + & + & + & + & + & + & Good \\
\hline Christensen et $a l^{12}$ & $\mathrm{RCT}$ & + & + & $?$ & - & - & - & + & + & + & + & + & Good \\
\hline Fukuda et $a l^{10}$ & $\mathrm{RCT}$ & + & + & $?$ & $?$ & + & + & - & + & + & + & - & Good \\
\hline Kinikli et $\left.a\right|^{33}$ & $\mathrm{RCT}$ & + & $?$ & + & - & - & + & $?$ & $?$ & + & + & - & Questionable \\
\hline Koutras et $a l^{36}$ & ССТ & - & - & + & + & + & + & + & - & + & + & - & Good \\
\hline Melikoglu et $a l^{37}$ & CCT & - & - & ? & $?$ & $?$ & + & $?$ & ? & + & + & - & Poor \\
\hline Salmon et $\left.\mathrm{a}\right|^{38}$ & СCT & - & - & $?$ & $?$ & ? & $?$ & - & $?$ & + & + & + & Questionable \\
\hline Sastre et $\left.a\right|^{34}$ & $\mathrm{RCT}$ & + & + & $?$ & $?$ & ? & + & + & + & + & + & + & Good \\
\hline Treacy et $a^{39}$ & СCT & - & - & $?$ & $?$ & ? & + & ? & ? & + & + & - & Poor \\
\hline Vadalà et $a l^{35}$ & $\mathrm{RCT}$ & + & + & ? & ? & ? & + & + & ? & + & + & - & Good \\
\hline
\end{tabular}

$\mathrm{CCT}$, clinical controlled trial; $\mathrm{RCT}$, randomised controlled trial. 
Table 3 Risk of bias assessment of cohort and cross-sectional studies

\begin{tabular}{|c|c|c|c|c|c|c|c|c|c|c|c|c|}
\hline Article & $\begin{array}{l}\text { Study } \\
\text { design }\end{array}$ & 1 & 2 & 3 & 4 & 5 & 6 & 7 & 8 & $\begin{array}{l}\text { Accurate } \\
\text { description } \\
\text { rehab. }\end{array}$ & $\begin{array}{l}\text { Men- } \\
\text { women ratio }\end{array}$ & Total score \\
\hline Åhlén et $a l^{54}$ & $\mathrm{RC}$ & + & - & + & + & $?$ & + & + & - & + & + & Good \\
\hline Ali et $\left.a\right|^{51}$ & CS & + & - & + & + & $?$ & $?$ & - & $?$ & + & - & Questionable \\
\hline Biernat et $a l^{40}$ & PC & - & - & - & - & $?$ & $?$ & $?$ & - & + & + & Poor \\
\hline Boszotta et al ${ }^{41}$ & $\mathrm{PC}$ & - & - & + & + & $?$ & $?$ & $?$ & - & - & $?$ & Poor \\
\hline Clark et $a l^{52}$ & CS & + & - & + & + & $?$ & + & $?$ & $?$ & - & + & Questionable \\
\hline Czamara et $a l^{42}$ & $\mathrm{PC}$ & + & - & + & + & $?$ & + & + & $?$ & + & + & Good \\
\hline Czaplicki et al ${ }^{46}$ & $\mathrm{PC}$ & + & - & + & + & $?$ & + & $?$ & - & - & - & Questionable \\
\hline Hill et $a l^{43}$ & PC & - & - & + & + & $?$ & + & - & $?$ & - & - & Poor \\
\hline Howell et $a l^{21}$ & $\mathrm{PC}$ & + & - & + & + & $?$ & + & + & $?$ & + & - & Good \\
\hline Janssen et $a l^{9}$ & CS & + & - & + & + & $?$ & + & + & + & + & $?$ & Good \\
\hline Janssen et al ref44 & PC & - & + & + & + & $?$ & + & + & + & + & - & Good \\
\hline Jenny et $a l^{47}$ & $\mathrm{PC}$ & - & + & + & + & $?$ & + & + & - & - & + & Good \\
\hline Karikis et $a l^{48}$ & $\mathrm{PC}$ & + & - & + & + & $?$ & + & + & - & - & + & Good \\
\hline Koutras et $a /^{49}$ & PC & + & - & + & + & - & + & + & - & - & - & Questionable \\
\hline Królikowska et al ${ }^{53}$ & CS & + & - & + & + & $?$ & - & + & - & + & - & Questionable \\
\hline Srinivas et $a l^{50}$ & PC & + & - & + & + & $?$ & + & + & - & - & - & Questionable \\
\hline Toanen et $\left.a\right|^{56}$ & $\mathrm{RC}$ & + & - & + & + & $?$ & + & + & - & - & + & Questionable \\
\hline Trojani et $a l^{55}$ & $\mathrm{RC}$ & + & + & + & + & ? & + & + & $?$ & + & - & Good \\
\hline Zaffagnini et $\left.a\right|^{45}$ & PC & + & - & + & + & - & + & + & + & - & + & Good \\
\hline
\end{tabular}

CS, cross-sectional study; PC, prospective cohort study; RC, retrospective cohort study; rehab., rehabilitation.

No differences were found between the two groups for IKDC, range of motion (ROM) and peak isometric force at 12 weeks postsurgery. ${ }^{12}$

Fukuda $e t a l^{10}$ evaluated the outcome of early start of open kinetic chain exercises in a restricted ROM at 1 year after non-anatomical, four-strand hamstring ACLR. A start of open kinetic chain quadriceps exercises at 4 weeks postoperatively in a restricted $\mathrm{ROM}\left(90^{\circ}-45^{\circ}\right)$ did not differ from a start at 12 weeks in terms of anterior knee laxity, pain and functional improvement. The early start group showed a faster recovery for quadriceps strength (19 weeks vs 17 months). ${ }^{10}$

The effect of progressive eccentric and concentric training at 12 weeks on functional performance after four-strand hamstring ACLR was investigated by Kinikli et $a l^{33}$ Outcome measures were isokinetic muscle strength, single and vertical hop tests, Lysholm score and ACL Quality of Life Questionnaire. There was a significant improvement of all outcome measures except for isokinetic strength of knee extensors and flexors. ${ }^{33}$

Baltaci $e t a l^{2}$ compared a 12-week Nintendo Wii Fit versus a conventional accelerated, brace-free rehabilitation after hamstring ACLR. The two different 12-week physiotherapy programme had the same effect on muscle strength, dynamic balance and functional performance values. ${ }^{32}$ Clark et $a \tilde{l}^{2}$ used the Nintendo Wii Fit Balance Board to assess weight-bearing asymmetry during squatting as outcome after hamstring autograft ACLR with accelerated rehabilitation. The authors found significant increases in asymmetry after ACLR compared with a matched control group. ${ }^{52}$

Jenny et $a l^{47}$ assessed functional outcome (sport activity, Tegner, Lysholm and IKDC subjective score) and rerupture rate after patient-based decision to return to work and sports. Return to work was possible for $96 \%$ of patients after a mean delay of 2.3 months. Return to sports was $92 \%, 6.1$ months for pivoting sports and 6.6 months for contact sports. A $6 \%$ rerupture rate occurred after a new significant knee injury. ${ }^{47}$ Assessing time to return to sports based on muscle strength may also be influenced by testing technique. Koutras $e t a t^{9}$ compared knee flexion isokinetic strength deficits between seated and prone positions after hamstring autograft ACLR with accelerated rehabilitation. Peak torque knee flexion deficits were higher in the prone position compared with the conventional seated position by an average of $6.5 \%$ at $60^{\circ} / \mathrm{s}$ and $9.1 \%$ at $180^{\circ} / \mathrm{s}$ $(\mathrm{p}<0.001)$. At 9 months after hamstring ACLR, most athletes would not be cleared to return to sports if tested in prone position. ${ }^{49}$

Brace-free accelerated rehabilitation after hamstring tendon autograft ACLR, early start of open kinetic chain quadriceps exercises at 4 weeks in a limited knee ROM $\left(90^{\circ}-45^{\circ}\right)$ and progressive concentric and eccentric exercises from 12 weeks do not alter clinical outcomes ('moderate' level of evidence). 


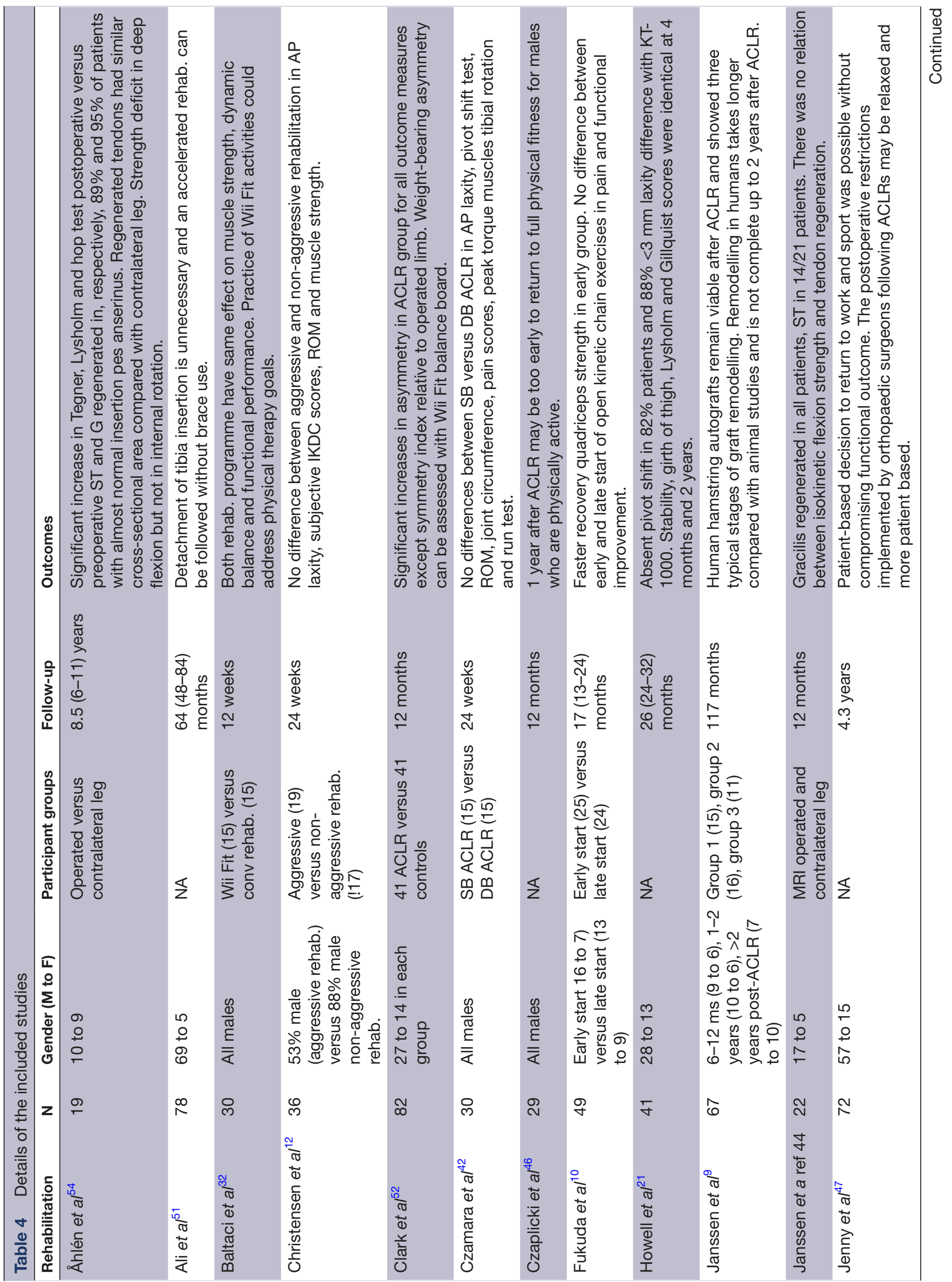




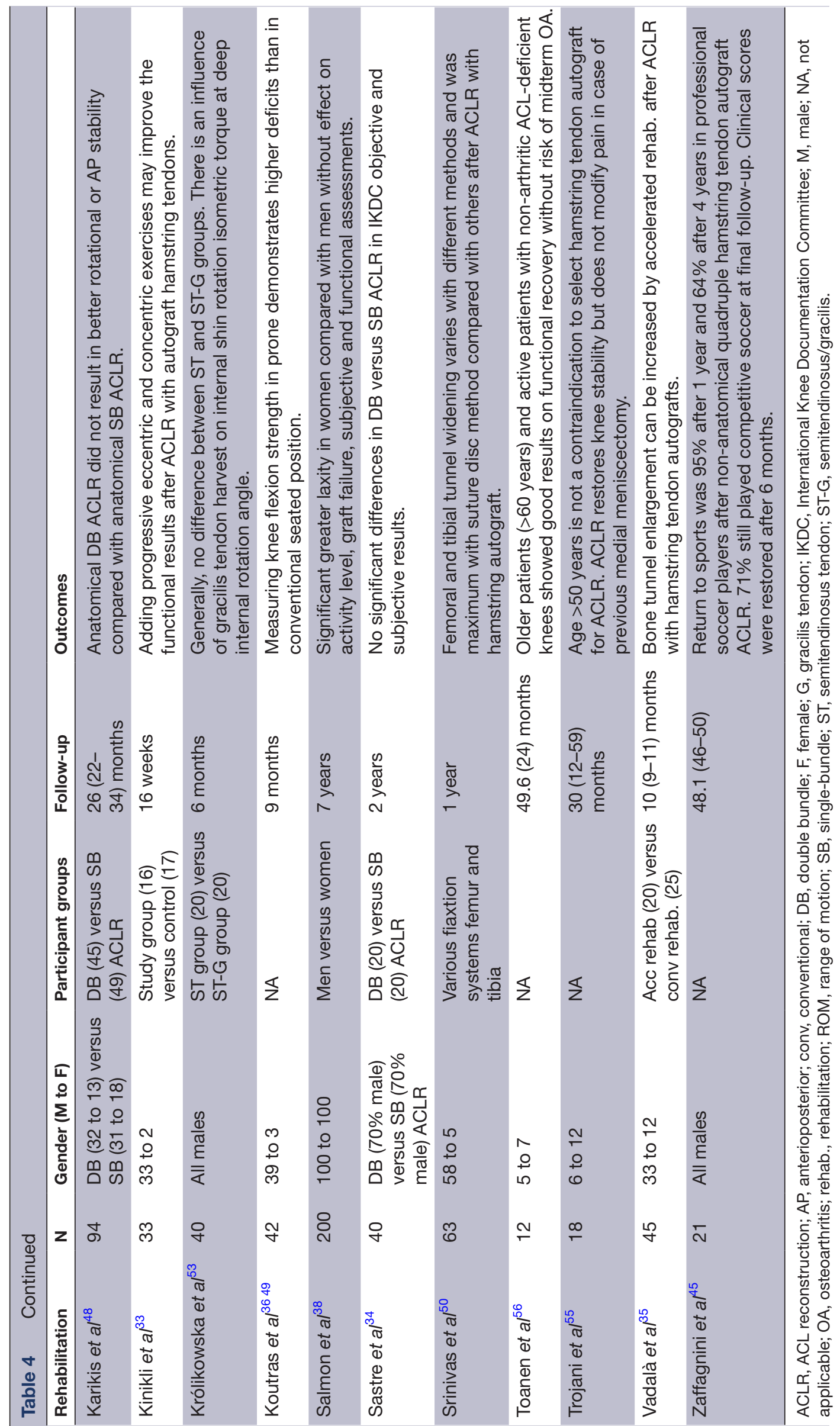




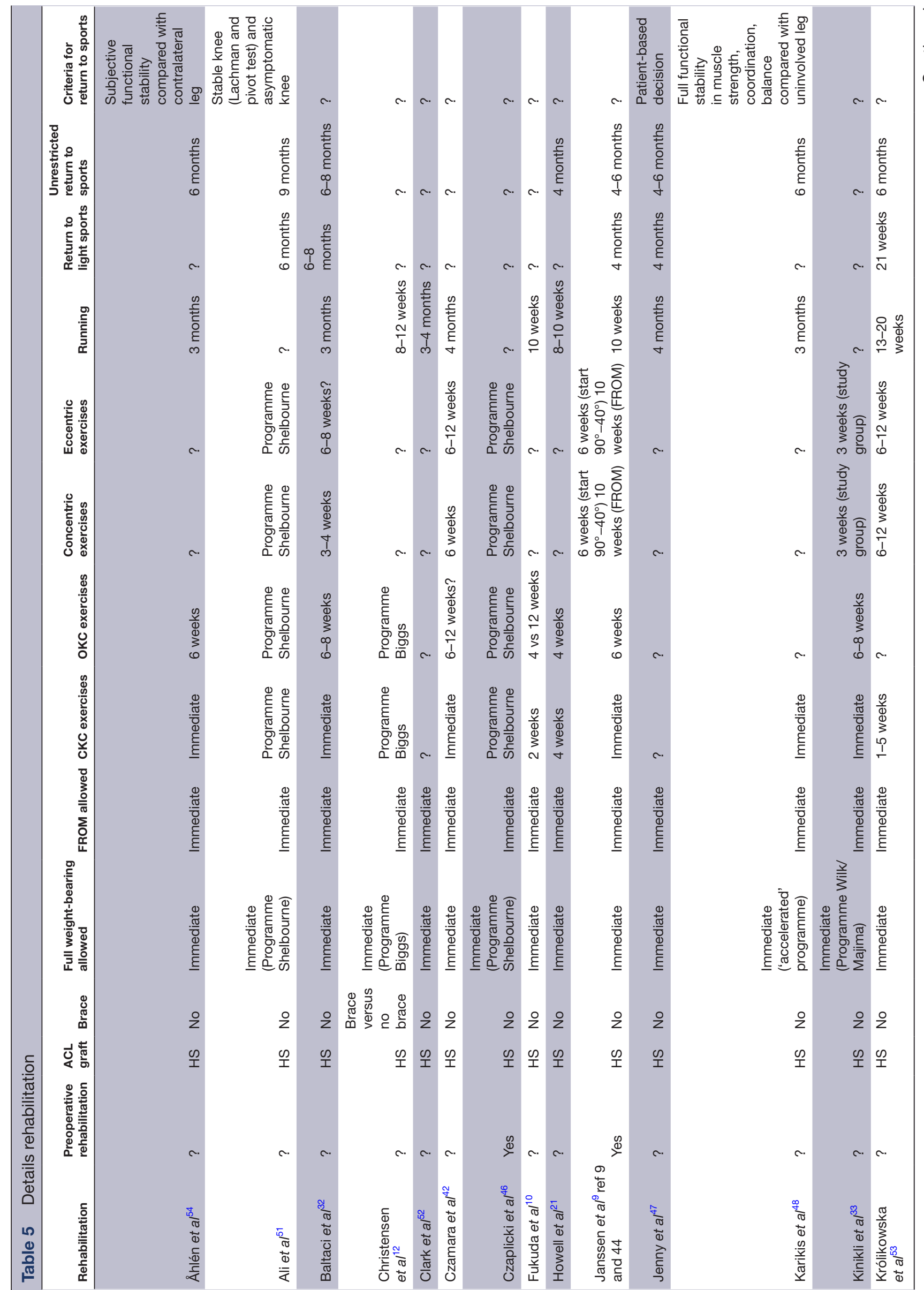




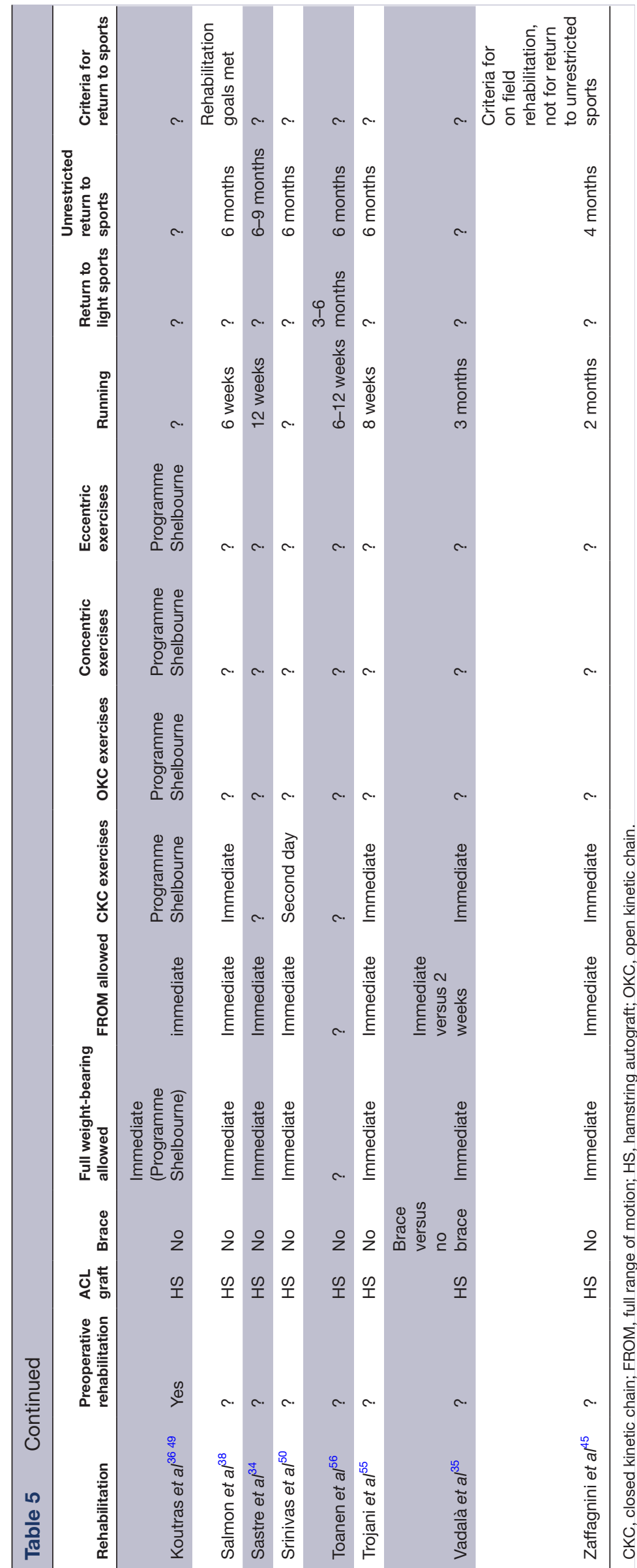


Isokinetic extension peak torque deficit is still present at 1 year after accelerated rehabilitation. The use of Nintendo Wii Fit activities could address weight-bearing asymmetry and physical therapy goals ('limited' level of evidence).

Patient-based decision to return to work and sports is possible without compromising functional outcome ('limited' level of evidence).

Measuring knee flexion strength in prone position shows larger knee flexion isokinetic deficits compared with the conventional seated position ('limited' level of evidence).

How do different patient characteristics affect clinical outcomes after hamstring tendon autograft ACLR with accelerated, bracefree rehabilitation?

Gender

Salmon $e t a \hat{l}^{8}$ did not find significant gender differences for graft rupture, activity level, self-reported or functional assessment or radiological outcome. Women did have significantly greater laxity than men on the Lachman test, pivot shift test and KT-1000 mean manual maximum testing at all time points. The higher laxity measurements did not influence the self-reported and functional outcome assessments. ${ }^{38}$

Gender does not influence clinical outcomes after hamstring tendon autograft ACLR with accelerated, brace-free, rehabilitation ('limited' level of evidence).

\section{Age}

Trojani et $a \tilde{l}^{\tilde{5}}$ retrospectively analysed the same ACLR technique as Salmon $e t a l^{88}$ in patients $>50$ years. Surgery restored knee stability but did not modify pain in patients with previous medial meniscectomy. Graft failure did not occur. The authors concluded that age over 50 years is not a contraindication to select a hamstring autograft for ACLR. ${ }^{55}$ Toanen et $a \tilde{l}^{56}$ demonstrated that older and active patients $>60$ years without osteoarthritis showed good results after single-bundle hamstring autograft ACLR. The majority of patients (83\%) returned to sports activities with $50 \%$ returning to their preinjury level of activity. ${ }^{56}$

Age $>50$ years does not influence clinical outcome after hamstring tendon autograft ACLR with accelerated, brace-free rehabilitation ('limited' level of evidence).

How do different non-anatomical and anatomical surgical techniques of hamstring tendon autograft ACLR affect clinical outcomes after accelerated, brace-free rehabilitation? Non-anatomical single tunnel four-strand hamstring tendon autograft ACLR

Three studies have examined this surgical technique. ${ }^{214551}$

Howell $e t a l^{21}$ presented a single surgeon prospective cohort series of transtibial ACLR technique with special attention to intercondylar roof impingement. Patients returned to unrestricted sports and work activities after 4 months. The authors justified the early return to vigorous activities at 4 months by unchanged knee stability, girth of the thigh, knee extension as well as Lysholm and Gillquist scores at 2-year follow-up. ${ }^{21}$

Ali $e t a b^{51}$ presented the outcomes of a single surgeon, cross-sectional study of transtibial non-anatomical ACLR using a hamstring graft without detachment of its tibial insertion. Follow-up was 64 (range 48-84) months. All patients achieved full ROM with a mean KT-1000 sideto-side difference of 1.43 (SD 3.86) and negative pivot shift test. The authors concluded that their technique showed satisfactory and comparable results to studies with conventional detachment of hamstring tendons from their tibial insertion. ${ }^{51}$

Zaffagnini et $a t^{45}$ analysed return to sports in a homogeneous group of male professional soccer players after ACLR. Follow-up was 4 years. The authors used a non-anatomical, four-strand hamstring technique with additional extra-articular fixation of the graft. After 12 months, $95 \%$ of patients returned to the preoperative professional soccer level. Mean time from surgery to first official match was 186 (range 107-282) days. The Knee injury and Osteoarthritis Outcome Scores reached the plateau level at 6 months postoperatively. At 4 years, $71 \%$ still played professional soccer, $62 \%$ at the same preoperative level and $9 \%$ in a lower division. Five per cent of patients experienced rerupture of the ACLR. ${ }^{45}$

Non-anatomical transtibial four-strand hamstring ACLR with accelerated, brace-free rehabilitation is associated with good clinical results. Return to sports was possible at 4-6 months postsurgery ('moderate' level of evidence).

\section{Non-anatomical versus anatomical hamstring tendon autograft $A C L R$}

Koutras et $a l^{36}$ compared the short-term functional and clinical outcomes between a non-anatomical transtibial versus an anatomical anteromedial ACL technique. The anteromedial approach group had better Lysholm scores at 3 months and better performance in the timed lateral movement functional tests at 3 and 6 months. All other comparisons were non-significant. ${ }^{36}$

Anatomical ACLR shows better short-term results than non-anatomical ACLR after accelerated, brace-free rehabilitation ('limited' level of evidence).

\section{Single-bundle versus double-bundle hamstring tendon autograft ACLR}

Sastre $e t a \ell^{34}$ compared anatomical four-strand singlebundle and double-bundle hamstring ACLR in a randomised prospective study. The authors did not find any difference between the two groups with respect to anterior laxity, pivot shift test, IKDC subjective and objective scores. ${ }^{34}$ In a similar study, Czamara $e t a l^{42}$ found no differences between the two groups for anterior tibial translation, pivot shift test, ROM and joint circumference, subjective assessment of pain and knee joint stability, peak torque for internal and external rotation and run test with maximal speed and change of direction manoeuvres. ${ }^{42}$ Karikis et al found that anatomical 
double-bundle ACLR did not result in better rotational or anteroposterior stability measurements than anteromedial portal non-anatomical single-bundle reconstruction at 2-year follow-up. ${ }^{48}$

There is no difference in clinical results between single-bundle and double-bundle hamstring tendon autograft ACLR with accelerated, brace-free rehabilitation ('strong' level of evidence).

\section{Semitendinosus versus combined semitendinosus/gracilis autograft ACLR}

Krolikowska $e t a \tilde{l}^{33}$ assessed isometric and peak torque of muscles responsible for internal and external rotation of the lower leg post-ACLR after a 6-month accelerated brace-free rehabilitation programme. There was no difference between patients reconstructed with only the semitendinosus autograft (ST) compared with patients reconstructed with a combined semitendinosus/gracilis autograft (STGR). There was, however, a significant difference in isometric internal rotation strength in the operated knee compared with the uninvolved knee at $25^{\circ}$ of internal rotation in the STGR group. ${ }^{53}$

There is an influence of additional gracilis harvest in internal rotation strength at a deep internal rotation angle ('limited' level of evidence).

Does accelerated, brace-free rehabilitation after hamstring tendon autograft ACLR influence tunnel widening?

Vadalà $e$ t $a l^{35}$ analysed tunnel widening after four-strand hamstring tendon ACLR by means of CT scan comparing accelerated brace-free rehabilitation versus non-accelerated rehabilitation with brace. Mean follow-up was 10 months. There was a significant increase in femoral and tibial tunnel diameter after accelerated, brace-free rehabilitation. ${ }^{35}$ The extend of tunnel widening with hamstring autograft and accelerated brace-free rehabilitation was measured by Srinivas et $a \check{l}^{0}$ with CT at 1-year follow-up: femoral and tibial tunnel widening varied with different methods of fixation and was maximal in the tibia with suture disc method compared with interference screw fixation. ${ }^{50}$

Accelerated, brace-free, rehabilitation after hamstring tendon autograft ACLR causes increased tunnel widening on both the femur and tibia ('limited' level of evidence).

Do hamstring tendons regenerate after harvest for ACLR with accelerated, brace-free rehabilitation?

Regeneration of hamstring tendons in the upper leg after harvest for ACLR with accelerated, bracefree rehabilitation was examined in two studies. ${ }^{44} 54$ Ahlen et $a \tilde{l}^{4}$ performed a retrospective MRI study with 6 -year follow-up after hamstring tendon harvest. The gracilis tendon regenerated in 18 of 19 patients, the ST tendon in 17 of 19 patients. ${ }^{54}$ Janssen et al ${ }^{44}$ performed a prospective MRI study in 22 patients with follow-up at 6 and 12 months. Regeneration of the gracilis tendon occurred in all patients, the ST tendon regenerated in 14 of 22 patients. The majority of tendons regenerated distal to the joint line of the knee. The authors did not find a significant relationship between isokinetic flexion strength and tendon regeneration. ${ }^{44}$

Hamstring tendons regenerate after harvest for ACLR. There is no evidence to support a relationship between increased isokinetic flexion strength and tendon regeneration ('strong' level of evidence).

Does the current biological knowledge of the hamstring graft support early return to sports after ACLR with accelerated, bracefree rehabilitation?

Janssen et at examined 67 midsubstance biopsies after clinically successful four-strand hamstring autograft ACLR with a standardised accelerated rehabilitation programme. Cellular density and vascular density were increased up to 24 months after ACLR. Especially the strong increase in myofibroblast density, from 13 to 24 months, indicated an active remodelling process from 1 to 2 years. Furthermore, vessel density increased over 24 months, whereas cell and myofibroblast density decreased but stayed higher than native hamstring and ACL controls. Collagen orientation did not return to normal in the study period. The authors question whether early return to sports (4-6 months) after accelerated rehabilitation is to be recommended after hamstring ACLR. ${ }^{9}$

Intra-articular hamstring graft remodelling is still active at 2 years after ACLR with an accelerated, bracefree rehabilitation. Based on the current evidence, the early return to sports after 4-6 months may be questionable ('limited' level of evidence).

\section{DISCUSSION}

A significant body of literature has shown that accelerated rehabilitation-defined as early-unrestricted motion, immediate weight-bearing and eliminating the use of immobilising braces-is appropriate after ACLR with patellar tendon grafts. ${ }^{12-20}$ However, conclusions are unclear when evaluating the effects of this type of rehabilitation after hamstring autograft ACLR. There are several factors that need to be considered. First, hamstring autografts require fixation of soft tissue (tendon) to bone. ${ }^{57} \mathrm{~A}$ period of 8-12 weeks is necessary for proper incorporation of hamstring grafts in the bone tunnels. ${ }^{58}$ Fixation of this soft tissue graft is considered the 'weak link' early on after ACLR. ${ }^{589}$ In a systematic review, Han et al concluded that both intratunnel and extratunnel fixation methods of hamstring ACL autografts displayed comparable outcomes based on objective IKDC, Lysholm and Tegner scores, anterior knee laxity and return to sports timing. ${ }^{59}$

Second, the intra-articular remodelling of the ACL hamstring autograft requires an optimal equilibrium between muscle strength training and graft loading to prevent stretch out of the ACL graft. ${ }^{2} 11152760$ Finally, early after ACLR, relative protection of the autograft donor site must be considered. Therefore, force generation from the hamstrings should be minimised when a hamstring autograft is employed. ${ }^{58}$ In summary, 
accelerated, brace-free rehabilitation needs to restore knee function and at the same time stimulate optimal graft healing. ${ }^{61}$

\section{Accelerated rehabilitation}

This review presented a 'moderate' level of evidence that accelerated rehabilitation after hamstring ACLR does not alter clinical outcome compared with non-accelerated rehabilitation with knee brace. ${ }^{12}$ The rationale of using a knee brace is to protect the healing graft during the early phases of rehabilitation. ${ }^{23}$ Various systematic reviews could not substantiate this hypothesis based on clinical results. ${ }^{23242862}$ Furthermore, full weight-bearing without crutches within 10 days (with a normal gait pattern) improves quadriceps function, prevents patellofemoral pain and does not affect knee stability. ${ }^{24} 62$

This review showed that start of open kinetic chain quadriceps exercises with $90^{\circ}-45^{\circ} \mathrm{ROM}$ at 4 weeks postsurgery does not alter the clinical outcome after hamstring autograft ACLR ('moderate' level of evidence). The combination of closed and open kinetic chain exercises protects the healing graft as a result of better dynamic lower extremity stability and neuromuscular control. ${ }^{61}$ Beynnon et $a l^{63}$ found similar maximum ACL strain values produced by active flexion-extension (an open kinetic chain exercise) and squatting (a closed kinetic chain exercise). They also demonstrated that increasing resistance during the squat exercise did not produce a significant increase in native ACL strain values, unlike increased resistance during active flexion-extension exercise. ${ }^{63}$ Escamilla $e t a b^{58}$ showed that non-weight-bearing exercises generally loaded the ACL graft more than weight-bearing exercises and that, for both exercises, the ACL was loaded to a greater extent between $10^{\circ}$ and $50^{\circ}$ compared with $50^{\circ}$ and $100^{\circ}$ of knee flexion. ${ }^{58}$ These biomechanical findings are in agreement with the good clinical results presented in this review with the early start of open kinetic exercises in a limited ROM. ${ }^{1064}$ Majima et $a l^{26}$ demonstrated that accelerated rehabilitation with open kinetic exercises started at 7-10 days after hamstring ACLR could rapidly restore muscle strength without significantly compromising graft stability. However, the incidence of synovitis of the knee was significantly increased after accelerated rehabilitation. ${ }^{26}$ Van Grinsven et al concluded in their systematic review on evidence-based rehabilitation after ACLR that there is increasing consensus that open kinetic chain exercises did not increase graft laxity (in and exceeding the safe range with a focus on endurance). Additionally, these exercises had a favourable effect on quadriceps strength. ${ }^{24}$

This review also demonstrated that start of eccentric and concentric muscle training at 12 weeks after surgery did not influence clinical outcome after hamstring autograft ACLR ('moderate' level of evidence). Therapeutic exercises that emphasise eccentric gluteus maximus, quadriceps femoris and gastrocnemius-soleus activation can improve lower extremity muscular shock absorption, prevent knee reinjury, enhance athletic performance, help heal lower extremity musculotendinous injuries, increase bone mineral density and decrease fall risk. ${ }^{61}$ Further research is warranted to determine the best timing of introducing open kinetic exercises and safe amount of progressive resistance training after ACLR with hamstring autografts. ${ }^{2465}$

A critical remark is necessary when accelerated rehabilitation is discussed. There is little consensus in the literature about what composes an accelerated rehabilitation programme because few papers have described their protocol adequately. ${ }^{24}$ In this review, almost all included studies on accelerated, brace-free rehabilitation agreed that immediate weight-bearing, full ROM and closed kinetic exercises were permitted after hamstring autograft ACLR. However, if even specified at all, the programme varied in their timing and details of open kinetic chain exercises, frequency of concentric and eccentric training and neuromuscular training (table 4). Few studies described full details of the accelerated rehabilitation after hamstring ACLR. The rehabilitation programme by Shelbourne and Nitz was most often cited. This programme emphasised specific presurgical rehabilitation goals. ${ }^{23} 242646$ 61-63 Remarkably, only five studies in this review provided specific details of this prehabilitation. ${ }^{93644649}$ Furthermore, although referring to the aforementioned rehabilitation protocols, the timing of return to activities such as running or unrestricted sports varied widely among studies, often without specific criteria (table 4). The lack of details of accelerated rehabilitation programme after hamstring autograft ACLR makes it difficult to evaluate the potential disadvantages of accelerated rehabilitation such as tunnel widening ${ }^{3566}$ and increased synovitis. ${ }^{26}$ Postoperative rehabilitation is a major factor contributing to the success of ACLR and needs to be defined in detail for adequate research on clinical outcome and safe return to sports.

\section{Return to sports}

Return to sports is often used as short-term to midterm outcome measure for ACLR and rehabilitation. ${ }^{25}$ In their meta-analysis of 69 articles, Ardern et a ${ }^{67}$ have shown that after ACLR, the overall return to some kind of sports activity is $81 \% .{ }^{67}$ Sixty-five per cent of patients returned to their preinjury level and $55 \%$ to competitive sports at final follow-up. ${ }^{67}$ Younger age, male gender and a positive psychological response all favoured returning to the preinjury level sport. ${ }^{67}$ Elite athletes had more than twice the odds of returning to competitive sports compared with non-elite athletes. ${ }^{67}$ This is supported by the evidence in the present review with $95 \%$ return to sports 1 year after with accelerated, brace-free rehabilitation. ${ }^{45}$ Elite male UEFA soccer league players needed 7 months to return to the first training after ACLR, 10 months to return to regular practice and 12 months to return to match play. ${ }^{68} 69$ Grindem et al have shown that the return to play after 9 months postsurgery substantially reduces ACL graft rerupture rate. ${ }^{70}$ Leading ACL 
experts generally let their patients return to sports at 6 months in average and involvement in active competition at 8 months postsurgery. ${ }^{71}$ However, a recent study by Herbst et al showed that most patients, in terms of neuromuscular abilities and compared with healthy controls, were most likely not ready for a safe return to sports, even 8 months postoperatively. The most limiting factor was a poor Limb symmetry index (LSI) value of $<90 \%$ if the dominant leg was involved and $<80 \%$ if the non-dominant leg was involved. ${ }^{72}$ Gokeler et al found that the majority of patients who are 6 months after ACLR require additional rehabilitation to pass return to sports criteria. ${ }^{73}$ Further studies identifying sport-specific differences in ACR outcomes in athletes could further enhance accelerated rehabilitation programme for athletes after ACLR. ${ }^{45} 73$

Graft failure after ACLR is not uncommon even with improved ACLR techniques. ${ }^{2}{ }^{3}$ Evidence-based evaluations did not prove a 4-6 months return to sports to be safe due to the fact that biological healing is not complete. $^{29} 69$ 74-76 This is also demonstrated in the current review: intra-articular hamstring graft remodelling was still active at 2 years after ACLR with an accelerated, brace-free rehabilitation. ${ }^{9}$ This may provide biological support for the findings by Paterno et al that in the 24 months after ACLR and return to sports, patients are at greater risk to suffer a subsequent ACL injury compared with young athletes without a history of ACL injuries. ${ }^{77}$ Considering the fact that rehabilitation protocols were extrapolated from animal in vivo studies, studies on human in vivo graft healing suggest a need for new postoperative rehabilitation schedules after ACLR with hamstring autografts. ${ }^{2}$ No final conclusions can be drawn on the mechanical strength of the healing ACL grafts in humans without any available technique for in vivo measurements of their mechanical properties. ${ }^{274}$

In this systematic review, only $20 \%$ of studies reported assessment criteria for return to sports after hamstring autograft ACLR. These criteria, however, lacked specific details for use in clinical practice or comparative scientific research. This is in agreement with previous reviews on return to sports after ACLR. ${ }^{28} 7879$ Furthermore, commonly used muscle functional tests are not demanding or sensitive enough to identify differences between injured and non-injured sides. ${ }^{69} 80$ Large meta-analysis have shown that despite $90 \%$ of patients having normal validated outcome scores, only $44 \%$ of patients returned to competitive sports. ${ }^{79} 81$ Currently, there are no concrete guidelines that allow for a safe return to unrestricted activity. ${ }^{79} 82$ Further research is necessary to develop a validated set of criteria to determine safe return to sport-specific training and unrestricted activity. ${ }^{6167697883}$

One of the strengths of this systematic review is that it presents all available knowledge on outcomes after hamstring tenson autograft ACLR with accelerated rehabilitation. This extensive search strategy was performed in several databases, for all relevant papers to be included. Furthermore, the PRISMA standard was applied to study selection, data collection, risk of bias assessment and reporting of results. This led to an extensive and complete overview of the current evidence on this topic with defined levels of evidence. As such, it is a useful paper for ACL experts in various fields of healthcare (eg, orthopaedic surgeons, physical therapists) and may facilitate interprofessional patient care. This systematic review also has limitations. Studies of different evidence levels were included in the search for all available knowledge on clinical outcome after accelerated, brace-free rehabilitation after ACLR. It must be noticed that the type of rehabilitation was not a primary intervention in all of the included studies. Some conclusions of the 'best-evidence synthesis' may therefore not be primarily related to accelerated rehabilitation. Another limitation is the inclusion of studies with limited number of patients. Furthermore, the 'best-evidence synthesis' by van Tulder $e t a l^{31}$ for this review may have limited the level of evidence due to the quality and limited number of studies for specific research questions. Although strict and adapted for various study types, the risk of bias assessment of the Cochrane Library may limit the strength of evidence. It may be argued that a 'low' risk of bias RCT study is of higher level of evidence than a 'low' risk of bias prospective cohort study.

The inclusion of merely publications in English is another limitation.

\section{CONCLUSIONS}

After hamstring tendon autograft ACLR with accelerated brace-free rehabilitation, clinical outcome is similar after single-bundle and double-bundle ACLR. Early start of open kinetic exercises at 4 weeks in a limited ROM $\left(90^{\circ}-45^{\circ}\right)$ and progressive concentric and eccentric exercises from 12 weeks postsurgery do not alter clinical outcome. Further research should focus on achievement of best balance between graft loading and graft healing in the various rehabilitation phases after ACLR as well as on validated, criterion-based assessments for safe return to sports.

Contributors All authors contributed to the search, screening, data collection, bias assessment and final writing of the manuscript.

Funding The authors have not declared a specific grant for this research from any funding agency in the public, commercial or not-for-profit sectors.

Competing interests None declared.

Patient consent Not required.

Provenance and peer review Not commissioned; externally peer reviewed.

Data sharing statement We are willing to share any further details of this PRISMA systematic review.

Open Access This is an Open Access article distributed in accordance with the Creative Commons Attribution Non Commercial (CC BY-NC 4.0) license, which permits others to distribute, remix, adapt, build upon this work non-commercially, and license their derivative works on different terms, provided the original work is properly cited and the use is non-commercial. See: http://creativecommons.org/ licenses/by-nc/4.0/

(C) Article author(s) (or their employer(s) unless otherwise stated in the text of the article) 2018. All rights reserved. No commercial use is permitted unless otherwise expressly granted. 


\section{REFERENCES}

1. Janssen RP. Anterior cruciate ligament reconstruction \& accelerated rehabilitation. Hamstring tendons, remodelling and osteoarthritis [PhD thesis]. Maastricht, The Netherlands: Maastricht University, 2016.

2. Janssen RP, Scheffler SU. Intra-articular remodelling of hamstring tendon grafts after anterior cruciate ligament reconstruction. Knee Surg Sports Traumatol Arthrosc 2014;22:2102-8.

3. Ménétrey J, Duthon VB, Laumonier T, et al. "Biological failure" of the anterior cruciate ligament graft. Knee Surg Sports Traumatol Arthrosc 2008;16:224-31.

4. van Eck CF, Schreiber VM, Mejia HA, et al. "Anatomic" anterior cruciate ligament reconstruction: a systematic review of surgical techniques and reporting of surgical data. Arthroscopy 2010;26(9 Suppl):S2-12.

5. Janssen RP, van Melick N, van Mourik JB. Similar clinical outcome between patellar tendon and hamstring tendon autograft after anterior cruciate ligament reconstruction with accelerated, bracefree rehabilitation: a systematic review. JISAKOS 2017. doi:10.1136/ jisakos-2016-000110. [Epub ahead of print 15 Sep 2017].

6. Kim HS, Seon JK, Jo AR. Current trends in anterior cruciate ligament reconstruction. Knee Surg Relat Res 2013;25:165-73.

7. Samuelsson K, Andersson D, Ahldén M, et al. Trends in surgeon preferences on anterior cruciate ligament reconstructive techniques. Clin Sports Med 2013;32:111-26.

8. Marumo K, Saito M, Yamagishi T, et al. The "ligamentization" process in human anterior cruciate ligament reconstruction with autogenous patellar and hamstring tendons: a biochemical study. Am J Sports Med 2005;33:1166-73.

9. Janssen RP, van der Wijk J, Fiedler A, et al. Remodelling of human hamstring autografts after anterior cruciate ligament reconstruction. Knee Surg Sports Traumatol Arthrosc 2011;19:1299-306.

10. Fukuda TY, Fingerhut D, Moreira VC, et al. Open kinetic chain exercises in a restricted range of motion after anterior cruciate ligament reconstruction: a randomized controlled clinical trial. Am J Sports Med 2013;41:788-94.

11. Scheffler SU, Unterhauser FN, Weiler A. Graft remodeling and ligamentization after cruciate ligament reconstruction. Knee Surg Sports Traumatol Arthrosc 2008;16:834-42.

12. Christensen JC, Goldfine LR, West HS. The effects of early aggressive rehabilitation on outcomes after anterior cruciate ligament reconstruction using autologous hamstring tendon: a randomized clinical trial. J Sport Rehabil 2013;22:191-201.

13. De Carlo M, Klootwyk TE, Shelbourne KD. ACL surgery and accelerated rehabilitation: revisited. J Sport Rehabil 1997;6:144-56.

14. Risberg MA, Holm I, Myklebust G, et al. Neuromuscular training versus strength training during first 6 months after anterior cruciate ligament reconstruction: a randomized clinical trial. Phys Ther 2007:87:737-50.

15. Beynnon BD, Johnson RJ, Naud S, et al. Accelerated versus nonaccelerated rehabilitation after anterior cruciate ligament reconstruction: a prospective, randomized, double-blind investigation evaluating knee joint laxity using roentgen stereophotogrammetric analysis. Am J Sports Med 2011;39:2536-48.

16. Shelbourne KD, Nitz P. Accelerated rehabilitation after anterior cruciate ligament reconstruction. Am J Sports Med 1990;18:292-9.

17. Biggs A, Jenkins WL, Urch SE, et al. Rehabilitation for patients following ACL reconstruction: a knee symmetry model. N Am J Sports Phys Ther 2009;4:2-12.

18. Shelbourne KD, Klotz C. What I have learned about the ACL utilizing a progressive rehabilitation scheme to achieve total knee symmetry after anterior cruciate ligament reconstruction. J Orthop Sci 2006;11:318-25.

19. Shelbourne KD, Vanadurongwan B, Gray T. Primary anterior cruciate ligament reconstruction using contralateral patellar tendon autograft. Clin Sports Med 2007;26:549-65.

20. Chapman A, Chamberlain V, Railton R, et al. Extensor strength in the anterior cruciate reconstructed knee. Aust J Physiother 1995;41:83-8

21. Howell SM, Taylor MA. Brace-free rehabilitation, with early return to activity, for knees reconstructed with a double-looped semitendinosus and gracilis graft. J Bone Joint Surg Am 1996;78:814-25

22. Shelbourne KD, Klootwyk TE, Decarlo MS. Update on accelerated rehabilitation after anterior cruciate ligament reconstruction. J Orthop Sports Phys Ther 1992;15:303-8.

23. Andersson D, Samuelsson K, Karlsson J. Treatment of anterior cruciate ligament injuries with special reference to surgical technique and rehabilitation: an assessment of randomized controlled trials. Arthroscopy 2009;25:653-85
24. van Grinsven S, van Cingel RE, Holla CJ, et al. Evidence-based rehabilitation following anterior cruciate ligament reconstruction. Knee Surg Sports Traumatol Arthrosc 2010;18:1128-44.

25. Wright RW, Preston E, Fleming BC, et al. A systematic review of anterior cruciate ligament reconstruction rehabilitation: part I: continuous passive motion, early weight bearing, postoperative bracing, and home-based rehabilitation. J Knee Surg 2008;21:217-24.

26. Majima T, Yasuda $\mathrm{K}$, Tago $\mathrm{H}$, et al. Rehabilitation after hamstring anterior cruciate ligament reconstruction. Clin Orthop Relat Res 2002;397:370-80.

27. Warner SJ, Smith MV, Wright RW, et al. Sport-specific outcomes after anterior cruciate ligament reconstruction. Arthroscopy 2011;27:1129-34.

28. Kruse LM, Gray B, Wright RW. Rehabilitation after anterior cruciate ligament reconstruction: a systematic review. J Bone Joint Surg Am 2012;94:1737-48.

29. Liberati A, Altman DG, Tetzlaff J, et al. The PRISMA statement for reporting systematic reviews and meta-analyses of studies that evaluate health care interventions: explanation and elaboration. J Clin Epidemiol 2009;62:e1-34.

30. Slavin RE. Best evidence synthesis: an intelligent alternative to meta-analysis. J Clin Epidemiol 1995;48:9-18.

31. van Tulder M, Furlan A, Bombardier C, et al. Updated method guidelines for systematic reviews in the cochrane collaboration back review group. Spine 2003;28:1290-9.

32. Baltaci G, Harput G, Haksever B, et al. Comparison between Nintendo Wii Fit and conventional rehabilitation on functional performance outcomes after hamstring anterior cruciate ligament reconstruction: prospective, randomized, controlled, double-blind clinical trial. Knee Surg Sports Traumatol Arthrosc 2013;21:880-7.

33. Kınıklı Gl, Yüksel I, Baltacı G, et al. The effect of progressive eccentric and concentric training on functional performance after autogenous hamstring anterior cruciate ligament reconstruction: a randomized controlled study. Acta Orthop Traumatol Turc 2014;48:283-9.

34. Sastre S, Popescu D, Núñez M, et al. Double-bundle versus singlebundle ACL reconstruction using the horizontal femoral position: a prospective, randomized study. Knee Surg Sports Traumatol Arthrosc 2010;18:32-6.

35. Vadalà A, lorio R, De Carli A, et al. The effect of accelerated brace free, rehabilitation on bone tunnel enlargement after ACL reconstruction using hamstring tendons: a CT study. Knee Surg Sports Traumatol Arthrosc 2007:15:365-71.

36. Koutras G, Papadopoulos P, Terzidis IP, et al. Short-term functional and clinical outcomes after $A C L$ reconstruction with hamstrings autograft: transtibial versus anteromedial portal technique. Knee Surg Sports Traumatol Arthrosc 2013;21:1904-9.

37. Melikoglu MA, Balci N, Samanci N, et al. Timing of surgery and isokinetic muscle performance in patients with anterior cruciate ligament injury. J Back Musculoskelet Rehabil 2008;21:23-8.

38. Salmon LJ, Refshauge KM, Russell VJ, et al. Gender differences in outcome after anterior cruciate ligament reconstruction with hamstring tendon autograft. Am J Sports Med 2006;34:621-9.

39. Treacy $\mathrm{SH}$, Barron OA, Brunet ME, et al. Assessing the need for extensive supervised rehabilitation following arthroscopic ACL reconstruction. Am J Orthop 1997;26:25-9.

40. Biernat R, Wołosewicz M, Tomaszewski W. A protocol of rehabilitation after $\mathrm{ACL}$ reconstruction using a hamstring autograft in the first month after surgery - a preliminary report. Ortop Traumatol Rehabil 2007;9:178-86.

41. Boszotta H. Arthroscopic reconstruction of anterior cruciate ligament using BTB patellar ligament in the press-fit technique. Surg Technol Int 2003;11:249-53.

42. Czamara A, Królikowska A, Szuba Ł, et al. Single- vs. double-bundle anterior cruciate ligament reconstruction: a new aspect of knee assessment during activities involving dynamic knee rotation. $J$ Strength Cond Res 2015;29:489-99.

43. Hill GN, O'Leary ST. Anterior cruciate ligament reconstruction: the short-term recovery using the Knee Injury and Osteoarthritis Outcome Score (KOOS). Knee Surg Sports Traumatol Arthrosc 2013;21:1889-94.

44. Janssen RP, van der Velden MJ, Pasmans HL, et al. Regeneration of hamstring tendons after anterior cruciate ligament reconstruction. Knee Surg Sports Traumatol Arthrosc 2013;21:898-905.

45. Zaffagnini S, Grassi A, Marcheggiani Muccioli GM, et al. Return to sport after anterior cruciate ligament reconstruction in professiona soccer players. Knee 2014;21:731-5.

46. Czaplicki A, Jarocka M, Walawski J. Isokinetic identification of knee joint torques before and after anterior cruciate ligament reconstruction. PLoS One 2015;10:e0144283. 
47. Jenny JY, Clement X. Patient-based decision for resuming activity after ACL reconstruction: a single-centre experience. Eur J Orthop Surg Traumatol 2016;26:929-35.

48. Karikis I, Ahldén M, Casut A, et al. Comparison of outcome after anatomic double-bundle and antero-medial portal non-anatomic single-bundle reconstruction in ACL-injured patients. Knee Surg Sports Traumatol Arthrosc 2017;25:1307-15.

49. Koutras G, Bernard M, Terzidis IP, et al. Comparison of knee flexion isokinetic deficits between seated and prone positions after ACL reconstruction with hamstrings graft: Implications for rehabilitation and return to sports decisions. J Sci Med Sport 2016;19:559-62.

50. Srinivas DK, Kanthila M, Saya RP, et al. Femoral and tibial tunnel widening following anterior cruciate ligament reconstruction using various modalities of fixation: a prospective observational study. J Clin Diagn Res 2016;10:RC09-11.

51. Ali MS, Kumar A, Adnaan Ali S, et al. Anterior cruciate ligament reconstruction using hamstring tendon graft without detachment of the tibial insertion. Arch Orthop Trauma Surg 2006;126:644-8.

52. Clark RA, Howells B, Feller J, et al. Clinic-based assessment of weight-bearing asymmetry during squatting in people with anterior cruciate ligament reconstruction using Nintendo Wii Balance Boards. Arch Phys Med Rehabil 2014;95:1156-61.

53. Królikowska A, Czamara A, Kentel M. Does gracilis tendon harvest during ACL reconstruction with a hamstring autograft affect torque of muscles responsible for shin rotation? Med Sci Monit 2015;21:2084-93.

54. Åhlén M, Lidén M, Bovaller Å, et al. Bilateral magnetic resonance imaging and functional assessment of the semitendinosus and gracilis tendons a minimum of 6 years after ipsilateral harvest for anterior cruciate ligament reconstruction. Am J Sports Med 2012;40:1735-41.

55. Trojani C, Sané JC, Coste JS, et al. Four-strand hamstring tendon autograft for ACL reconstruction in patients aged 50 years or older. Orthop Traumatol Surg Res 2009;95:22-7.

56. Toanen C, Demey G, Ntagiopoulos PG. Is there any benefit in anterior cruciate ligament reconstruction in patients older than 60 years? Am J Sports Med 2016;363546516678723.

57. Muller B, Bowman KF, Bedi A. ACL graft healing and biologics. Clin Sports Med 2013;32:93-109.

58. Escamilla RF, Macleod TD, Wilk KE, et al. Anterior cruciate ligament strain and tensile forces for weight-bearing and non-weight-bearing exercises: a guide to exercise selection. J Orthop Sports Phys Ther 2012;42:208-20.

59. Han DL, Nyland J, Kendzior M, et al. Intratunnel versus extratunnel fixation of hamstring autograft for anterior cruciate ligament reconstruction. Arthroscopy 2012;28:1555-66.

60. Beynnon BD, Fleming BC, Johnson RJ, et al. Anterior cruciate ligament strain behavior during rehabilitation exercises in vivo. Am J Sports Med 1995;23:24-34.

61. Nyland J, Brand E, Fisher B. Update on rehabilitation following ACL reconstruction. Open Access J Sports Med 2010;1:151-66.

62. Wright RW, Preston E, Fleming BC, et al. A systematic review of anterior cruciate ligament reconstruction rehabilitation: part II: open versus closed kinetic chain exercises, neuromuscular electrical stimulation, accelerated rehabilitation, and miscellaneous topics. J Knee Surg 2008;21:225-34.

63. Beynnon BD, Johnson RJ, Fleming BC, et al. The strain behavior of the anterior cruciate ligament during squatting and active flexionextension. A comparison of an open and a closed kinetic chain exercise. Am J Sports Med 1997;25:823-9.

64. Janssen RP, du Mée AW, van Valkenburg J, et al. Anterior cruciate ligament reconstruction with 4-strand hamstring autograft and accelerated rehabilitation: a 10-year prospective study on clinical results, knee osteoarthritis and its predictors. Knee Surg Sports Traumatol Arthrosc 2013;21:1977-88.
65. Adams D, Logerstedt DS, Hunter-Giordano A, et al. Current concepts for anterior cruciate ligament reconstruction: a criterionbased rehabilitation progression. J Orthop Sports Phys Ther 2012;42:601-14.

66. Clatworthy MG, Annear P, Bulow JU, et al. Tunnel widening in anterior cruciate ligament reconstruction: a prospective evaluation of hamstring and patella tendon grafts. Knee Surg Sports Traumatol Arthrosc 1999;7:138-45

67. Ardern CL, Taylor NF, Feller JA, et al. Fifty-five per cent return to competitive sport following anterior cruciate ligament reconstruction surgery: an updated systematic review and meta-analysis including aspects of physical functioning and contextual factors. $\mathrm{Br} J$ Sports Med 2014:48:1543-52.

68. Waldén M, Hägglund M, Magnusson H, et al. Anterior cruciate ligament injury in elite football: a prospective three-cohort study. Knee Surg Sports Traumatol Arthrosc 2011;19:11-19.

69. Renström PA. Eight clinical conundrums relating to anterior cruciate ligament $(A C L)$ injury in sport: recent evidence and a personal reflection. Br J Sports Med 2013;47:367-72.

70. Grindem H, Snyder-Mackler L, Moksnes H, et al. Simple decision rules can reduce reinjury risk by $84 \%$ after $A C L$ reconstruction: the Delaware-Oslo ACL cohort study. Br J Sports Med 2016;50:804-8.

71. Middleton KK, Hamilton T, Irrgang JJ, et al. Anatomic anterior cruciate ligament (ACL) reconstruction: a global perspective. Part 1. Knee Surg Sports Traumatol Arthrosc 2014;22:1467-82.

72. Herbst E, Hoser C, Hildebrandt C, et al. Functional assessments for decision-making regarding return to sports following $A C L$ reconstruction. Part II: clinical application of a new test battery. Knee Surg Sports Traumatol Arthrosc 2015;23:1283-91.

73. Gokeler A, Welling W, Zaffagnini S, et al. Development of a test battery to enhance safe return to sports after anterior cruciate ligament reconstruction. Knee Surg Sports Traumatol Arthrosc 2017;25:192-9.

74. Claes S, Verdonk P, Forsyth R, et al. The "ligamentization" process in anterior cruciate ligament reconstruction: What happens to the human graft? A systematic review of the literature. Am J Sports Med 2011;39:2476-83.

75. Pauzenberger L, Syré S, Schurz M. “Ligamentization” in hamstring tendon grafts after anterior cruciate ligament reconstruction: a systematic review of the literature and a glimpse into the future. Arthroscopy 2013;29:1712-21.

76. Tohyama H, Yoshikawa T, Ju YJ, et al. Revascularization in the tendon graft following anterior cruciate ligament reconstruction of the knee: its mechanisms and regulation. Chang Gung Med J 2009;32:133-9.

77. Paterno MV, Rauh MJ, Schmitt LC, et al. Incidence of second ACL injuries 2 years after primary ACL reconstruction and return to sport. Am J Sports Med 2014;42:1567-73.

78. Barber-Westin SD, Noyes FR. Factors used to determine return to unrestricted sports activities after anterior cruciate ligament reconstruction. Arthroscopy 2011;27:1697-705.

79. Harris JD, Abrams GD, Bach BR, et al. Return to sport after ACL reconstruction. Orthopedics 2014;37:e103-8.

80. Barber-Westin SD, Noyes FR. Objective criteria for return to athletics after anterior cruciate ligament reconstruction and subsequent reinjury rates: a systematic review. Phys Sportsmed 2011;39:100-10.

81. Ardern CL, Taylor NF, Feller JA, et al. Return-to-sport outcomes at 2 to 7 years after anterior cruciate ligament reconstruction surgery. Am J Sports Med 2012;40:41-8.

82. Yabroudi MA, Irrgang JJ. Rehabilitation and return to play after anatomic anterior cruciate ligament reconstruction. Clin Sports Med 2013;32:165-75.

83. Engelen-van Melick N, van Cingel RE, Tijssen MP, et al. Assessment of functional performance after anterior cruciate ligament reconstruction: a systematic review of measurement procedures. Knee Surg Sports Traumatol Arthrosc 2013;21:869-79. 